\title{
An Image Registration Approach to Automated Calibration for Freehand 3D Ultrasound
}

\author{
J.M. Blackall ${ }^{1}$, D. Rueckert ${ }^{2}$, C.R. Maurer, Jr. ${ }^{3}$, G.P. Penney ${ }^{1}$, D.L.G. Hill ${ }^{1}$, and \\ D.J. Hawkes ${ }^{1}$ \\ 1 Division of Radiological Sciences and Medical Engineering \\ The Guy's King's and St. Thomas' Schools of Medicine and Dentistry \\ Guy's Hospital, London SE1 9RT, UK \\ 2 Department of Computing, Imperial College, London SW7 2BZ, UK \\ 3 Department of Neurological Surgery and Biomedical Engineering \\ University of Rochester, Rochester, NY 14642, USA
}

\begin{abstract}
This paper describes an image registration approach to calibration for freehand three-dimensional (3D) ultrasound. If a conventional ultrasound probe is tracked using a position sensor, and the relation between this sensor and the two-dimensional (2D) image plane is known, the resulting set of B-scans may be correctly compounded into an image volume. Calibration is the process of determining the transformation (rotation, translation, and optionally image scaling) that maps B-mode image slice coordinates to points in the coordinate system of the tracking sensor mounted on the ultrasound probe. A set of $2 \mathrm{D}$ ultrasound images of a calibration phantom is obtained using a tracked ultrasound probe. Calibration is performed by searching for the calibration parameters that maximise the similarity between a model of the calibration phantom, which can be an image volume or a geometrical model, and the ultrasound images transformed into the coordinate space of the phantom. Validation of this calibration method is performed using a gelatin phantom. Measures of the calibration reproducibility, reconstruction precision and reconstruction accuracy are presented for this technique, and compared to those obtained using a conventional cross-wire phantom. Registration-based calibration is shown to be a rapid and accurate method of automatic calibration for freehand 3D ultrasound.
\end{abstract}

\section{Introduction}

Three-dimensional ultrasound addresses some of the disadvantages of conventional two-dimensional ultrasound imaging, reducing subjectivity inherent in the mental transformation of the 2D images into an anatomical volume; reducing the problems of speckle, shadowing and enhancement; and improving diagnostic (e.g., obstetrics) and therapeutic (e.g., biopsy, brachytherapy, cryosurgery, RF ablation) decisions [4]. One approach to $3 \mathrm{D}$ ultrasound uses a $2 \mathrm{D}$ phased array of elements, which are used to transmit a broad beam of ultrasound diverging away from the array and sweeping out a pyramidal volume. Another approach is to control the position of the transducer using stepping motors in the transducer's scan head or a translational or rotational mechanical device. The technique under consideration in this paper is that of sensed freehand acquisition. 
Freehand 3D ultrasound is based on tracking the arbitrary motion of a conventional B-mode probe. The motion of the probe in all six degrees of freedom is tracked using a sensor that provides position and orientation, which is commonly magnetic $[1,3,6,7]$, but may also be optical, mechanical or acoustic. The probe is moved slowly over the region of interest. The B-scans are digitised by a frame grabber, tagged with the measurements from the position sensor, and stored as 2D arrays of pixels. Providing that the probe has been calibrated, the set of B-scans may then be assembled into a 3D data set, using the associated position data. The scan planes may intersect each other so that the same region in space is sampled from more than one direction, but gaps may remain where no samples are taken. The data may then be visualised by reslicing, or by creating 3D surface or volume renderings [8].

To calibrate the ultrasound probe, the position and orientation of the B-scan plane with respect to the sensor mounted on the probe must be determined. The calibration transformation consists of six rigid-body transformation parameters (three for rotation and three for translation) and optionally two image scale factors. These are combined with the position sensor measurements to calculate the correct positions of the B-scans during reconstruction. Accurate calibration is essential for a consistent reconstruction that preserves true anatomical shape and must be repeated every time a sensor is mounted on a probe $[7,8]$.

An estimate of the six rigid-body calibration parameters can be obtained by external measurements of the probe and position sensor [5]. However, the origin of the sensor coordinate system is not well defined with respect to the external cases of the sensor and probe. Calibration is usually performed by imaging an artificial object with known physical properties or dimensions, known as a phantom. Measurements obtained by scanning the phantom, combined with its known physical properties, can be used to determine the six rigid body parameters and two scaling factors [8]. Several common approaches use an invariant point, line, or plane. An invariant point is typically obtained with the cross-wire phantom [1,3,7], but a small metal sphere can also be used [6]. Another calibration phantom is the three-wire phantom [2], which consists of three orthogonal wires mounted in a water bath. An invariant plane can be the floor of a water bath [7,8], or the "virtual plane' provided by the "Cambridge phantom" [7]. A comparison of existing calibration techniques has been carried out by Prager et al [7].

In this paper we propose a new calibration algorithm for freehand ultrasound images. The calibration algorithm is based on the assumption that correct calibration parameters should allow the registration of a set of tracked 2D ultrasound images of a phantom with a digital model of its structure in $3 \mathrm{D}$.

\section{Method}

The calibration algorithm is based on the assumption that the correct calibration parameters will provide the optimal registration of a set of tracked $2 \mathrm{D}$ ultrasound images of a phantom with a 3D model of the phantom, which can be an image volume generated using CT or MR, or a geometrical model. Figure 1 illustrates the four different coordinate systems used by the calibration algorithm. The ultrasound coordinate system describes points in the ultrasound image. The probe coordinate system describes points 
relative to a tracking sensor attached to the ultrasound probe. The world coordinate system forms the reference frame and describes points in physical space, i.e., relative to the origin of the tracking device. The transformation between probe coordinates and world coordinates is the output of tracking the position sensor relative to the origin of the tracking device. The model coordinate system describes points in model space. The transformation between model and world coordinates can be determined by attaching fiducial markers to the phantom.

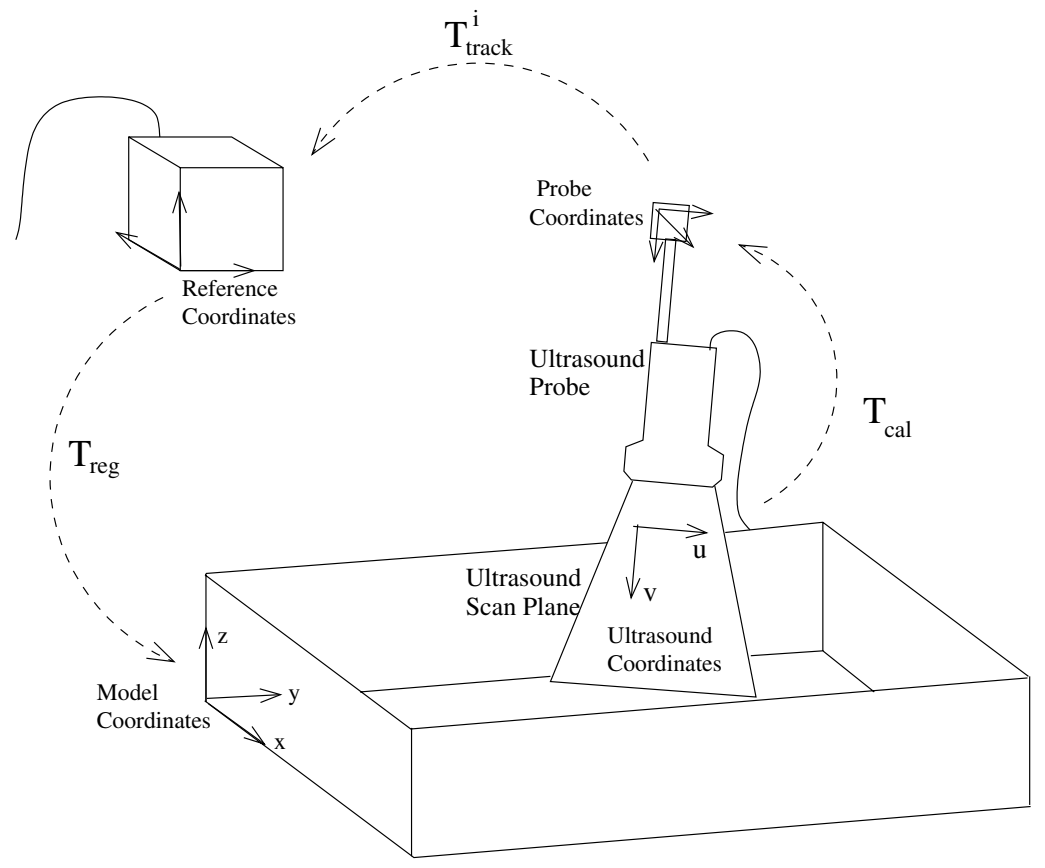

Fig. 1. Coordinate systems used in calibration algorithm.

The transformation between coordinates in the ultrasound image and coordinates in the model can be expressed as follows:

$$
\left(\begin{array}{c}
x_{i} \\
y_{i} \\
z_{i} \\
1
\end{array}\right)=\mathbf{T}_{r e g} \mathbf{T}_{\text {track }}^{i} \mathbf{T}_{c a l}\left(\begin{array}{c}
u_{i} \\
v_{i} \\
0 \\
1
\end{array}\right)
$$

where $x_{i}, y_{i}, z_{i}$ are model coordinates; $u_{i}, v_{i}$ are coordinates in the $i$ th ultrasound image; $\mathbf{T}_{\text {reg }}$ is the rigid-body registration transformation, from world to model coordinates; $\mathbf{T}_{\text {track }}^{i}$ is the tracking transformation, from probe to world coordinates, for the $i$ th image; and $\mathbf{T}_{c a l}$ is the rigid body (and optionally image scaling) calibration trans- 
formation, from ultrasound to probe coordinates. In this equation only the calibration transformation is unknown, all the other transformations are known.

\subsection{Similarity Measure}

A 3D digital model of the calibration phantom may be generated by imaging the phantom, e.g., in CT or MR. Since the properties of the imaging modality used to derive the model data may be quite different from those of ultrasound, the similarity measure must be able to deal with these. In this paper we use normalised mutual information (NMI) as a similarity measure which has been shown to align images from different modalities accurately and robustly and is invariant to the amount of overlap [11]:

$$
I(A, B)=\frac{H(A)+H(B)}{H(A, B)}
$$

Here $H(A), H(B)$ denote the marginal entropies of both images and $H(A, B)$ denotes their joint entropy. The marginal and joint entropies are both calculated from the joint histogram of $A$ and $B$. If the correct calibration parameters are found the ultrasound images and model images should be correctly aligned and the normalised mutual information should be maximal.

The similarity measure is calculated by taking each 2D ultrasound image in turn. For each pixel in the ultrasound image the corresponding location in the model data is calculated using equation (1) and the intensity of the model at this location is calculated from the eight neighbouring voxels using trilinear interpolation. The pixel intensity of the original ultrasound image and the interpolated model intensity are used to add a single value to the joint histogram, $H(A, B)$. This approach avoids compounding a 3D ultrasound image before evaluating the similarity measure, as would be necessary if a traditional image-to-image registration were performed.

\subsection{Optimisation}

Given an initial estimate of the calibration transformation $\mathbf{T}_{c a l}$, an improved estimate may be determined by finding the calibration transformation $\mathbf{T}$ which maximises the similarity measure $I$ as a function of the set of ultrasound images $B$ and the digital model $A$ :

$$
\hat{\mathbf{T}}_{c a l}=\arg \max _{\mathbf{T}}\{I(A, \mathbf{T}(B))\}
$$

In this paper we use a simple iterative optimisation algorithm. At each iteration the algorithm evaluates the similarity measure for the current estimate of the calibration parameters as well as for the current estimate with increments and decrements of step size $\pm \delta$. At the end of each iteration the current estimate of the calibration parameters is updated with those estimates which have maximised the similarity measure.

For computational efficiency we have implemented a multi-resolution search strategy which searches for the calibration parameters at different levels of image resolution and with different step sizes [11]. The algorithm starts with an initial step size of $\delta=4 \mathrm{~mm}$ for the translation parameters, $\delta=4^{\circ}$ for rotation parameters, and $\delta=4 \%$ 
for scale changes. If no further improvement of the similarity measure can be achieved, the step size is reduced by a factor of two and the process is repeated. The algorithm stops if the step size reaches a lower limit, having been halved eight times.

\section{Calibration Experiments}

The ultrasound machine used in our experiments is a Siemens Sonoline Versa Pro. Calibration experiments were performed on a $10 \mathrm{MHz}$ linear array probe (Siemens 10.0 L 25). A depth setting of $40 \mathrm{~mm}$ was used, and controls such as time-gain compensation, number of focal zones and overall gain were set appropriately and kept constant throughout all experiments. Twenty infrared LEDs were attached to the ultrasound probe. The probe was tracked during image acquisition using an optical tracking system (Optotrak 3020, Northern Digital) which tracks infrared LEDs to a 3D spatial accuracy of approximately $0.2 \mathrm{~mm}$.

\subsection{Calibration Phantoms}

Gelatin Phantom A gelatin phantom, pictured in Figure 2, was constructed with which to carry out registration-based calibration. This multimodality phantom was formed using gelatin powder (G-2500, Sigma Chemicals) dissolved in water at a concentration of $15 \%$ by weight. A $2 \%$ by weight concentration of silica (S-5631, Sigma Chemicals) was added to generate speckle texture and also provide MR contrast within included structures, as shown in Figure 3. These materials yield acoustic and mechanical properties which are similar to those of human tissue [9] and so allow correct image scaling factors to be determined. The phantom was constructed in several stages. A block of gelatin containing silica was allowed to set, then a selection of shapes were cut from this block, and placed onto a base layer of set gelatin in a rigid acrylic container. This was then covered with more molten gelatin, leaving the structures of interest embedded at various depths and orientations. An MR scan of the gelatin phantom was acquired to act as its digital model. Eight fiducial markers filled with a dilute aqueous solution of Gadolinium were attached to the acrylic container. The spatial resolution of the MR scan was $0.869 \mathrm{~mm} \times 0.869 \mathrm{~mm} \times 1.165 \mathrm{~mm}$.

Ten sets of calibration data, each consisting of 100 ultrasound images, were acquired with a layer of water covering the top of the phantom to provide acoustic coupling whilst allowing free movement of the probe. During scanning, an effort was made to move the probe in all six degrees of freedom while imaging the regions of interest. Prior to ultrasound image acquisition the fiducial markers were accurately localised using an Optotrak ball-tipped probe, allowing the registration transformation, $\mathbf{T}_{r e g}$, between physical and model coordinates to be determined. For each data set, the algorithm was tested with 64 starting estimates, based on a preliminary point-based calibration, but with all combinations of $\pm 5 \mathrm{~mm}$ and $\pm 5^{\circ}$ added to each of the six rigid-body parameters.

Cross-Wire Phantom Point-based calibration is a very widely used technique in freehand 3D ultrasound and has been shown to yield the most precise and accurate recon- 


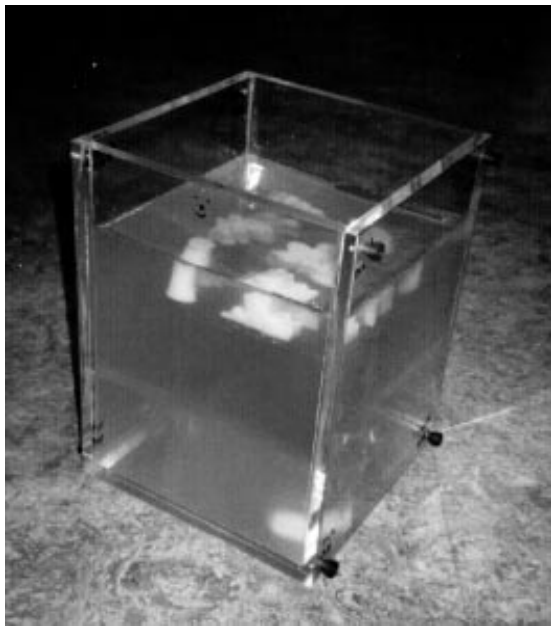

(a)

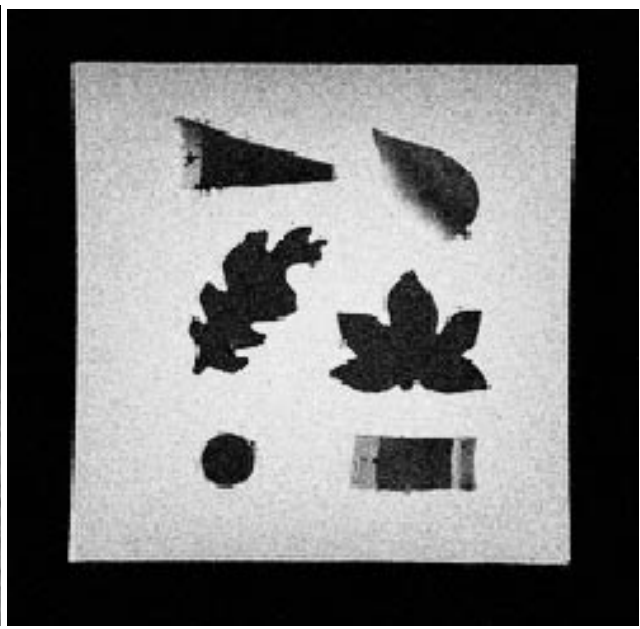

(b)

Fig. 2. (a) The gelatin phantom used for registration-based calibration experiments, (b) MR slice through gelatin phantom showing shapes of internal structures. Calibration is achieved by automatic registration of a set of tracked $2 \mathrm{D}$ ultrasound images to this MR image volume.

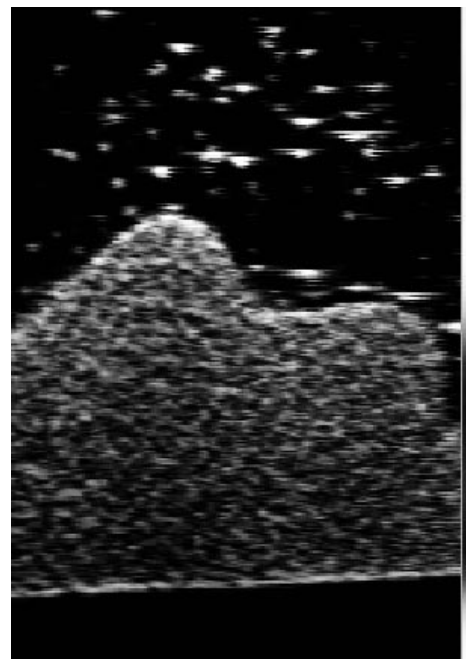

Fig. 3. Corresponding US and MR image slices through gelatin phantom. 
structions [7]. It is therefore useful to compare the point-based method to our registrationbased approach to calibration. A cross-wire phantom was constructed by crossing two cotton threads in a water bath, as described in $[1,3,7]$. Ten cross-wire data sets were then acquired, each consisting of 50 ultrasound images, and the point at which the wires cross was manually segmented from each.

\subsection{Assessment of Calibration Quality}

The quality of our registration-based calibration was assessed using several criteria, based on a previously published protocol [7], and compared to a conventional crosswire calibration.

Calibration Reproducibility Reproducibility of calibrations was assessed by looking at the position of a single point in the ultrasound image in probe coordinates under two different calibration transformations. The right corner furthest from the transducer was chosen to allow comparison with previously reported experiments [7]. The Euclidean distance between these two points in probe coordinates was taken as a measure of reproducibility, and this was calculated for each pair of calibrations.

$$
\Delta \mathbf{x}^{r}=\left\|\mathbf{T}_{\text {cal } 1} \mathbf{x}^{c}-\mathbf{T}_{\text {cal } 2} \mathbf{x}^{c}\right\|
$$

where $\Delta \mathrm{x}^{r}$ is the reproducibility measure; $\mathrm{x}^{c}$ is the corner point of the ultrasound image; and $\mathbf{T}_{\text {cal 1 }}, \mathbf{T}_{\text {cal } 2}$ are the two calibration transformations.

Reconstruction Precision Reconstruction precision was assessed by scanning a crosswire from 50 different viewpoints. The cross was manually located in each image and then transformed to reconstruction space using the associated tracking data, and the calibration on test. The change in 3D position was calculated as a measure of reconstruction precision. This was calculated for each pair of views, using all calibrations.

$$
\Delta \mathbf{x}^{p}=\left\|\mathbf{T}_{\text {track }}^{\text {view } 1} \mathbf{T}_{\text {cal }} \mathbf{x}^{\text {view } 1}-\mathbf{T}_{\text {track }}^{\text {view } 2} \mathbf{T}_{\text {cal }} \mathbf{x}^{\text {view } 2}\right\|
$$

where $\Delta \mathbf{x}^{p}$ is the precision measure; $\mathbf{T}_{\text {track }}$ view $^{i}$ is the tracking transformation corresponding to the $i$ th view of the cross-wire; $\mathbf{x}^{\text {view } i}$ is the $i$ th cross-wire point in ultrasound image coordinates; and $\mathbf{T}_{c a l}$ is the calibration transformation.

Reconstruction Accuracy Reconstruction accuracy was assessed by scanning 8 ball bearings, 10 times each. The centre of the ball was located in each image, then transformed to reconstruction space using the associated tracking data and the calibration on test. These ball bearings were $3 \mathrm{~mm}$ in diameter, and were set in $3 \mathrm{~mm}$ hemispherical divots, which were accurately machined in a sheet of acrylic. The divots were arranged in two rows of four, successive divots were separated by approximately $100 \mathrm{~mm}$. The position of the centre of each divot was determined using an Optotrak pointer with a $3 \mathrm{~mm}$ diameter ball-point tip and thus corresponds to centre of the ball bearing. The Euclidean distance between this gold-standard position and the position determined by 
manual identification in the ultrasound image was taken as a measure of point reconstruction accuracy, and was calculated for each view of each ball bearing, using all calibrations.

$$
\Delta \mathbf{x}^{a}=\left\|\mathbf{T}_{\text {track }}{ }^{\text {view } 1} \mathbf{T}_{\text {cal }} \mathbf{x}^{\text {view } 1}-\mathbf{x}^{g s}\right\|
$$

where $\Delta \mathbf{x}^{a}$ is the point reconstruction accuracy measure; $\mathbf{T}_{\text {track }}{ }^{\text {view }}{ }^{i}$ is the tracking transformation corresponding to a view of the $i$ th ball bearing; $\mathbf{x}^{\text {view } i}$ is the $i$ th ball bearing centre point in ultrasound image coordinates; $\mathbf{x}^{g s}$ is the gold-standard position of the centre of the divot; and $\mathbf{T}_{c a l}$ is the calibration transformation on test.

Similarly, the difference between the gold-standard separation and the separation determined from the ultrasound images was taken as a measure of distance reconstruction accuracy, and calculated for each pair of views of adjacent ball bearings, using all calibrations.

$$
\Delta \mathbf{x}^{d}=\left\|\left(\mathbf{T}_{\text {track }}^{\text {view } 1} \mathbf{T}_{\text {cal }} \mathbf{x}^{\text {view } 1}-\mathbf{T}_{\text {track }}^{\text {view } 2} \mathbf{T}_{\text {cal }} \mathbf{x}^{\text {view } 2}\right)\right\|-\left\|\left(\mathbf{x}^{g s 1}-\mathbf{x}^{g s 2}\right)\right\|
$$

where $\Delta \mathbf{x}^{d}$ is the distance reconstruction accuracy measure; $\mathbf{T}_{\text {track }}{ }^{\text {view }}{ }^{i}$ is the tracking transformation corresponding to The $i$ th view of a ball bearing; $\mathbf{x}^{\text {view } i}$ is the $i$ th ball bearing centre point in ultrasound image coordinates; $\mathbf{x}^{g s i}$ is the gold-standard position of the centre of the $i$ th divot; and $\mathbf{T}_{c a l}$ is the calibration transformation on test.

\section{Results}

The results of the calibration quality experiments are summarised in table 1 . The calibration reproducibility measure $\Delta \mathrm{x}^{r}$ indicates the extent to which repeated experiments using the same technique yield the same calibration parameters. The calibration precision measure $\Delta \mathrm{x}^{p}$ indicates the precision with which a single physical point can be reconstructed from a number of ultrasound images. The calibration point reconstruction accuracy measure $\Delta \mathrm{x}^{a}$ indicates the accuracy with which points can be reconstructed in the correct positions and similarly the distance reconstruction accuracy measure $\Delta \mathrm{x}^{d}$

\begin{tabular}{|c|c|c|c|c|c|c|c|c|}
\hline Quality Measure & \multicolumn{2}{|r|}{$\Delta \mathrm{x}^{r}$} & \multicolumn{2}{|r|}{$\Delta \mathrm{x}^{p}$} & \multicolumn{2}{|r|}{$\Delta \mathrm{x}^{a}$} & \multicolumn{2}{|c|}{$\Delta \mathrm{x}^{d}$} \\
\hline Calibration Method & Point & Registration & Point & Registration & Point & Registration & Point & Registration \\
\hline Mean $(\mathrm{mm})$ & 1.05 & 1.84 & 0.80 & 1.15 & 1.15 & 1.16 & -0.00019 & -0.025 \\
\hline Std. Dev. (mm) & 0.43 & 1.26 & 0.46 & 0.62 & 0.40 & 0.45 & 0.60 & 0.69 \\
\hline $\operatorname{Max}(\mathrm{mm})$ & 2.00 & 4.91 & 3.52 & 5.15 & 3.10 & 3.18 & 1.74 & 2.44 \\
\hline $\operatorname{Min}(\mathrm{mm})$ & 0.19 & 0.00 & 0.02 & 0.02 & 0.32 & 0.24 & -2.57 & -2.97 \\
\hline
\end{tabular}
indicates the extent to which distances can be correctly reconstructed.

Table 1. Summary of results of calibration validation experiments. Four measures of calibration quality, $\Delta \mathrm{x}^{r}, \Delta \mathrm{x}^{p}, \Delta \mathrm{x}^{a}$, and $\Delta \mathrm{x}^{d}$ are shown for both the conventional Point-based method and our Registration-based technique. 
These results indicate that the registration-based calibration approach yields similarly impressive point and distance accuracy results to the conventional point-based method. On average, both techniques allow point reconstruction to within just over $1 \mathrm{~mm}$ of the true position in world coordinates, and distance reconstruction to within $0.025 \%$. Point-based calibration performs slightly better than registration-based in terms of reproducibility and precision, tending to produce more stable calibration parameters, and more precise reconstructions, but in all quality measures the differences are fairly small.

\section{Discussion}

In this paper we have proposed a novel registration-based method for 3D calibration of a $2 \mathrm{D}$ ultrasound probe. The method entails establishing the 3D rigid-body transformation and 2 scaling factors between the 2D ultrasound image coordinates and the probe coordinates. This is achieved by scanning an object of known dimensions and finding the calibration transformation that maximises the normalised mutual information between a set of 2D ultrasound images and a 3D model of the scanned object.

We have shown that the accuracy, precision and reproducibility of registration-based calibration is comparable with that obtained using the established point-based method. Our new method has the significant advantage that it takes about 2 minutes to acquire the data, and subsequent processing of the data is fully automatic, requiring no segmentation. The optimisation algorithm currently takes about 15 minutes to run on a Sun Ultra 10 workstation, which is practical for most applications, but we believe that improvements to the algorithm could allow running time to be reduced even further. In comparison, point-based calibration requires about 30 minutes of careful scanning, followed by 30 minutes of user interaction with the data, to manually identify the crosswire point in each image.

The current method of phantom construction is sub optimal in that the gelatine used may change shape over time and may gain or lose water during the scanning process. Also, the MR image used as our 3D digital model of the phantom may also introduce errors due to geometric distortion and uncertainty in image scaling. If the geometry of the phantom was determined using a more accurate scan, for example CT, or an accurately machined ultrasound compatible phantom were constructed, we would expect a corresponding increase in accuracy, precision, and reproducibility.

Values of calibration quality measures resulting from our registration-based calibration experiments compare favourably with equivalent measures reported previously in other studies of calibration techniques for 3D freehand ultrasound [7]. However, these values are not directly comparable, due to the differences in accuracy of the tracking devices used in the two studies.

\section{Acknowledgements}

We would like to thank EPSRC for financial support, and the members of the Computational Imaging Science Group, for their assistance and encouragement. 


\section{References}

1. C. D. Barry, C. P. Allott, N. W. John, P. M. Mellor, P. A. Arundel, D. S. Thomson, and J. C. Waterton. Three-dimensional Freehand Ultrasound: Image Reconstruction and Volume Analysis. Ultrasound in Medicine and Biology, 23(8):1209-1224, 1997.

2. J. Carr. Surface Reconstruction in 3D Medical Imaging. PhD Thesis, University of Canterbury, Christchurch, New Zealand, 1996.

3. P. R. Detmer, G. Bashein, T. Hodges, K. W. Beach, E. P. Filer, D. H. Burns, and D. E. Strandness, Jr. 3D Ultrasonic Image Feature Localization based on Magnetic Scanhead Tracking: In Vitro Calibration and Validation. Ultrasound in Medicine and Biology, 20(4):923-936, 1994.

4. A. Fenster and D. B. Downey. 3D Ultrasonic Imaging: A Review. IEEE Engineering in Medicine and Biology, 15(6):41-51, 1996.

5. S. W. Hughes, T. J. D’Arcy, D. J. Maxwell, W. Chiu, A. Milner, J. E. Saunders, and R. J. Sheppard. Volume Estimation from Multiplanar 2D Ultrasound Images Using a Remote Electromagnetic Position and Orientation Sensor. Ultrasound in Medicine and Biology, 22(5):561-572, 1996.

6. D. F. Leotta, P. R. Detmer, and R. W. Martin. Performance of a Miniature Magnetic Position Sensor for Three-dimensional Ultrasound Imaging. Ultrasound in Medicine and Biology, 23(4):597-609, 1997.

7. R. W. Prager, R. N. Rohling, A. H. Gee, and L. Berman. Rapid Calibration for 3-D Freehand Ultrasound. Ultrasound in Medicine and Biology, 24(6):855-869, 1998.

8. R. N. Rohling. 3D Freehand Ultrasound: Reconstruction and Spatial Compounding. PhD Thesis, University of Cambridge, England, 1998.

9. L. K. Ryan and F. S. Foster. Tissue Equivalent Vessel Phantoms for Intravascular Ultrasound. Ultrasound in Medicine and Biology, 23(2):261-273, 1997.

10. C. Studholme, D. L. G. Hill, and D. J. Hawkes. Automated 3-D registration of MR and CT images of the head. Medical Image Analysis, 1(2):163-175, 1996.

11. C. Studholme, D. L. G. Hill, and D. J. Hawkes. An overlap invariant entropy measure of 3D medical image alignment. Pattern Recognition, 32(1):71-86, 1999. 